\title{
Biomarkers of oxidative stress following continuous positive airway pressure withdrawal: data from two randomised trials
}

\author{
John R. Stradling ${ }^{1}$, Esther I. Schwarz ${ }^{2}$, Christian Schlatzer ${ }^{2}$, Ari R. Manuel ${ }^{1}$, \\ Regent Lee ${ }^{3}$, Charalambos Antoniades ${ }^{3}$ and Malcolm Kohler ${ }^{2}$
}

Affiliations: ${ }^{1}$ Oxford Centre for Respiratory Medicine and NIHR Oxford Biomedical Research Centre, Churchill Hospital Campus, Oxford University, Oxford, UK. ${ }^{2}$ Sleep Disorders Centre and Pulmonary Division, University Hospital of Zurich, Zurich, Switzerland. ${ }^{3}$ Division of Cardiovascular Medicine, Radcliffe Department of Medicine, John Radcliffe Campus, University of Oxford, Oxford, UK.

Correspondence: John Stradling, Oxford Centre for Respiratory Medicine, Churchill Hospital Campus, Oxford OX3 7LJ, UK. E-mail: john.stradlingdorh.nhs.uk

ABSTRACT There is conflicting evidence whether intermittent hypoxia in obstructive sleep apnoea (OSA) influences oxidative stress. We hypothesised that withdrawal of continuous positive airway pressure (CPAP) from patients with OSA would raise markers of oxidative stress.

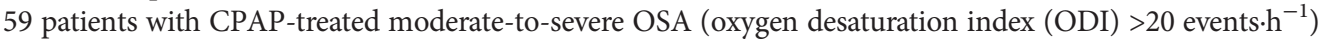
were randomised 1:1 to either stay on CPAP $(n=30)$ or change to sham CPAP $(n=29)$ for 2 weeks. Using samples from two similar studies at two sites, we measured early morning blood malondialdehyde (MDA, a primary outcome in one study and a secondary outcome in the other), lipid hydroperoxides, total antioxidant capacity, superoxide generation from mononuclear cells and urinary F2-isoprostane. We also measured superoxide dismutase as a marker of hypoxic preconditioning. "Treatment" effects (sham CPAP versus $\mathrm{CPAP}$ ) were calculated via linear regression.

Sham CPAP provoked moderate-to-severe OSA (mean ODI 46 events $\cdot \mathrm{h}^{-1}$ ), but blood markers of oxidative stress did not change significantly (MDA “treatment" effect $(95 \% \mathrm{CI})-0.02(-0.23$ to +0.19$\left.) \mu \mathrm{mol} \cdot \mathrm{L}^{-1}\right)$. Urinary F2-isoprostane fell significantly by $\sim 30 \%\left(-0.26(-0.42\right.$ to -0.10$\left.) \mathrm{ng} \cdot \mathrm{mL}^{-1}\right)$ and superoxide dismutase increased similarly $\left(+0.17(+0.02\right.$ to +0.30$\left.) \mathrm{ng} \cdot \mathrm{mL}^{-1}\right)$.

We found no direct evidence of increased oxidative stress in patients experiencing a return of their moderate-to-severe OSA. The fall in urinary F2-isoprostane and rise in superoxide dismutase implies that hypoxic preconditioning may have reduced oxidative stress.

@ERSpublications

Obstructive sleep apnoea may induce hypoxic preconditioning and reduce, rather than increase, oxidative stress http://ow.ly/MaUoN

This article has supplementary material available from erj.ersjournals.com

Received: Feb 102015 | Accepted after revision: April 102015 | First published online: June 112015

Support statement: Primary sources of funding: British Heart Foundation (PG/12/80/29891) and Swiss National Science Foundation (32003B_143365/1). Secondary sources of funding: Oxford Health Services Research Committee, Oxford Radcliffe Charitable Funds, National Institute for Health Research Oxford Biomedical Research Centre, based at Oxford University Hospitals NHS Trust and University of Oxford. Funding information for this article has been deposited with FundRef.

Clinical trial: This study is listed on the International Standard Randomized Controlled Trial Number Register (identifier number ISRCTN 73047833) and registered at www.clinicaltrials.gov (identifier number NCT 01797653).

Conflict of interest: Disclosures can be found alongside the online version of this article at erj.ersjournals.com

Copyright OERS 2015 


\section{Introduction}

Obstructive sleep apnoea (OSA) is a common problem and usually attended by oscillations in oxygen saturation. There is a clear association between OSA and endothelial dysfunction [1, 2], hypertension [3] and cardiovascular disease [4], but the potential mechanisms by which OSA may cause cardiovascular disease are still debated [5]. There is robust evidence from randomised controlled trials that increased catecholamine production occurs with OSA $[1,6]$. There is additional evidence that intermittent hypoxaemia, such as that observed in OSA, may provoke endothelial dysfunction [7] and therefore potentially the onset of atherosclerosis $[8,9]$.

The evidence for a link between intermittent hypoxaemia and vascular damage is contradictory [10, 11]. One proposed mechanism is that the repetitive oscillations in oxygen levels may provoke increased oxidative stress, via so-called ischaemia/reperfusion injury $[7,12,13]$. During periods of hypoxaemia, antioxidant mechanisms are downregulated, such that when oxygen levels rise again, more reactive oxygen species (ROS) or free radicals, with one or more unpaired electrons, are produced in mitochondria, in excess of that which can usually be quenched (by antioxidants such as superoxide dismutase). These excess ROS, made up of unpaired oxygen atoms $\left(\bullet \mathrm{O}_{2}^{-}\right.$, superoxide, primary ROS) or oxygen-containing molecules (secondary ROS, e.g. $\cdot \mathrm{OH})$, are then thought, among other things, to provoke systemic inflammation, endothelial damage and dysfunction: the path to atheroma [12]. There is also evidence that during hypoxic episodes, xanthine dehydrogenase is converted to xanthine oxidase; hypoxic neutrophils then consume excessive ATP with accumulation of purine catabolites (xanthine) which, following re-oxygenation, are metabolised by the increased xanthine oxidase, thus producing abnormal amounts of superoxide radical and hydrogen peroxide [14]. Part of the toxicity of primary ROS results from the generation of many further toxic molecules such as hydrogen peroxide and hydroxyl radicals (secondary ROS), and peroxynitrite (a reactive nitrogen species made from nitric oxide (NO) and superoxide). Endothelial function and vasodilation may thus be impaired, as it is dependent on NO. Increased levels of antioxidants have been shown to partially prevent the damage from ischaemia/reperfusion injury [14]. The majority of experimental evidence in support of this hypothesis comes from animal experiments, most of which used more severe and prolonged periods of intermittent hypoxaemia than are seen in patients with OSA [13-16]. Whether the short-cycle intermittent hypoxaemia observed in patients with OSA ( 1-min cycle lengths) activates any of these mechanisms is not clear, and the length and depth of the transient hypoxia may be critical $[17,18]$.

We have recently developed an experimental protocol to investigate the pathophysiology of human OSA [1]. In patients with OSA, already established on continuous positive airway pressure (CPAP), comparing 2 weeks of CPAP withdrawal (versus continuing CPAP) allows robust randomised controlled trials to be performed. Using this experimental protocol, we have investigated the hypothesis that 2 weeks of OSA will increase circulating markers of oxidative stress.

\section{Methods}

\section{Trial design}

These data come from two hospital-based randomised controlled trials with similar protocols, that allowed us to evaluate the effects of 14 days of CPAP withdrawal versus continuing CPAP on measures of oxidative stress in patients with prior CPAP-treated OSA. The design is similar to that reported in an earlier trial from the same units [1], except that in the current study patients had more severe OSA.

\section{Patients}

Patients previously diagnosed with OSA, and treated with CPAP, who were registered in a database of either the Oxford Centre for Respiratory Medicine (Churchill Hospital, Oxford, UK) or the Sleep Disorders Centre (University Hospital Zurich, Switzerland) were eligible for the trial if they were aged 20-75 years, had an oxygen desaturation index (ODI) $\left(\geqslant 4 \%\right.$ dips) of $>20$ events $\cdot h^{-1}$ during their original diagnostic sleep study, had been treated with CPAP for $>12$ months (with an average compliance of $\geqslant 4 \mathrm{~h}$ per night) and had a residual apnoea-hypopnoea index $<10$ events $\cdot \mathrm{h}^{-1}$ on CPAP. Such patients were contacted and asked to take part in the study, the first part of which was to establish that they had an ODI $>20$ events. $\mathrm{h}^{-1}$ during an initial pre-trial period without CPAP. Exclusion criteria are described in the online supplementary material.

\section{Intervention, randomisation and blinding}

Following the initial week of overnight oximetry ( 3 nights on CPAP to confirm efficacy, and 4 nights off CPAP to verify the return of sufficiently severe OSA prior to trial entry), the patients returned to automatic CPAP (S9 AutoSet, ResMed, Abingdon, UK) for a minimum of 2 weeks. Patients were computer-randomised 1:1 (with minimisation for OSA severity and body mass index (BMI)) (Minim, http://www-users.york.ac.uk/ mb55/guide/minim.htm) to either continue automatic CPAP or switch to subtherapeutic sham CPAP for 2 weeks. A description of sham CPAP construction, and the limited blinding achieved, is included in the online supplementary material. 
Sample preparation

The preparation of the samples for analysis is described in the online supplementary material.

\section{Outcome measures}

Continuing abolition of OSA by CPAP at baseline was further confirmed on a home (Oxford) or hospital (Zurich) sleep study the night prior to randomisation (Black Shadow (Stowood Scientific Instruments, Oxford, UK) or Alice5 (Philips Respironics AG, Zofingen, Switzerland)) and the severity of any OSA after 2 weeks was similarly assessed using a repeat sleep study. The additional measurements made, including blood pressure, are described in the online supplementary material. The main outcome of this analysis was the change in plasma malondialdehyde (MDA) levels over the 2 weeks in CPAP withdrawal subjects versus control subjects. In Oxford the primary outcome of the study was the change in plasma MDA levels, but in Zurich the identical protocol was run with a different primary outcome (hyperaemic myocardial blood flow); we took advantage of this and collected exactly similar samples to combine with the Oxford data. Secondary outcomes were plasma oxidised low-density lipoprotein, serum total antioxidant capacity, urinary F2-isoprostanes (fresh morning urine sample), and NADPH oxidase-derived superoxide generation from peripheral blood mononuclear cells.

\section{Measurement of circulating markers}

The laboratory techniques for measuring the markers of oxidative stress are described in the online supplementary material.

\section{Sample size and statistical analysis}

The approach to a sample size calculation is described in the online supplementary material. "Treatment" effects (CPAP withdrawal versus control) were modelled using linear regression (IBM SPSS, version 17; IBM, Portsmouth, UK), controlling for the baseline values of the relevant dependent variable, age, BMI, statins, antihypertensives and smoking status. The 95\% confidence intervals of the observed treatment effects reveal the actual power of the study for each of the primary and secondary end-points.

\section{Results}

59 patients were included in this study (Zurich $n=45$, Oxford $n=14$ ) and recruited between January 2013 and March 2014. The combination of samples from the two sites provided data from more subjects than required by the power calculation, thus allowing fewer numbers than intended to be recruited in Oxford. A full record of patients contacted, but who declined participation, was not kept. No subjects dropped out following randomisation. Figure 1 shows the subject flow chart. Baseline characteristics of the control and CPAP-withdrawal groups are shown in table 1. The two cohorts were well matched, with no suggestion of large enough differences to bias the results.

Consistent with our previous study, conducted under a similar protocol [1], in the CPAP-withdrawal group there was a significant increase in morning office systolic blood pressure $(+6.1 \mathrm{mmHg}, 95 \% \mathrm{CI}+0.6$ to +11.6; $\mathrm{p}=0.032)$, diastolic blood pressure $(+4.2 \mathrm{mmHg}, 95 \% \mathrm{CI}+0.04$ to $+8.4 ; \mathrm{p}=0.048)$ and heart rate $\left(+8.3\right.$ beats $\cdot \mathrm{min}^{-1}, 95 \% \mathrm{CI}+3.9$ to $\left.+12.5 ; \mathrm{p}<0.001\right)$, compared to the continuing-CPAP control group. Similarly, Epworth Sleepiness Scale scores rose $(+3.6$ points, $95 \%$ CI +2.0 to $+5.0 ; \mathrm{p}<0.001)$, compared to the continuing-CPAP control group.

Table 2 shows the results of the oxidative stress analyses. Despite the cardiovascular changes and a clear difference in the degree of intermittent hypoxia (ODI), there was no evidence of an increase in markers of oxidative stress. Interestingly, and contrary to our hypothesis, we observed a significant reduction in urinary F2-isoprostane in the CPAP-withdrawal patients compared to controls $(\mathrm{p}=0.002)$. In addition, there was a significant correlation between the ODI at 2 weeks and the fall in F2-isoprostanes $(r=-0.41, n=59$, $\mathrm{p}=0.001)$.

\section{Discussion}

This is the first randomised controlled study to look at the effect of 2 weeks of CPAP withdrawal and consequent intermittent hypoxia on markers of oxidative stress in patients with OSA. By selecting patients with moderate-to-severe OSA for entry into the CPAP-withdrawal model, we were able to induce a clear difference in the degree of intermittent hypoxia between the two groups (mean $\geqslant 4 \% 46$ ODI events.h ${ }^{-1}$ versus 3 events. $\mathrm{h}^{-1}$ at 2 weeks). In addition there was a clear and large effect on both systolic and diastolic blood pressure, as well as heart rate. Despite these substantial cardiovascular changes after 2 weeks of nocturnal intermittent hypoxia, we did not observe increased markers of oxidative stress. On the contrary, the unexpected reduction in one marker, the urinary F2-isoprostanes, implied a lessening of oxidative stress, which seems counterintuitive. 


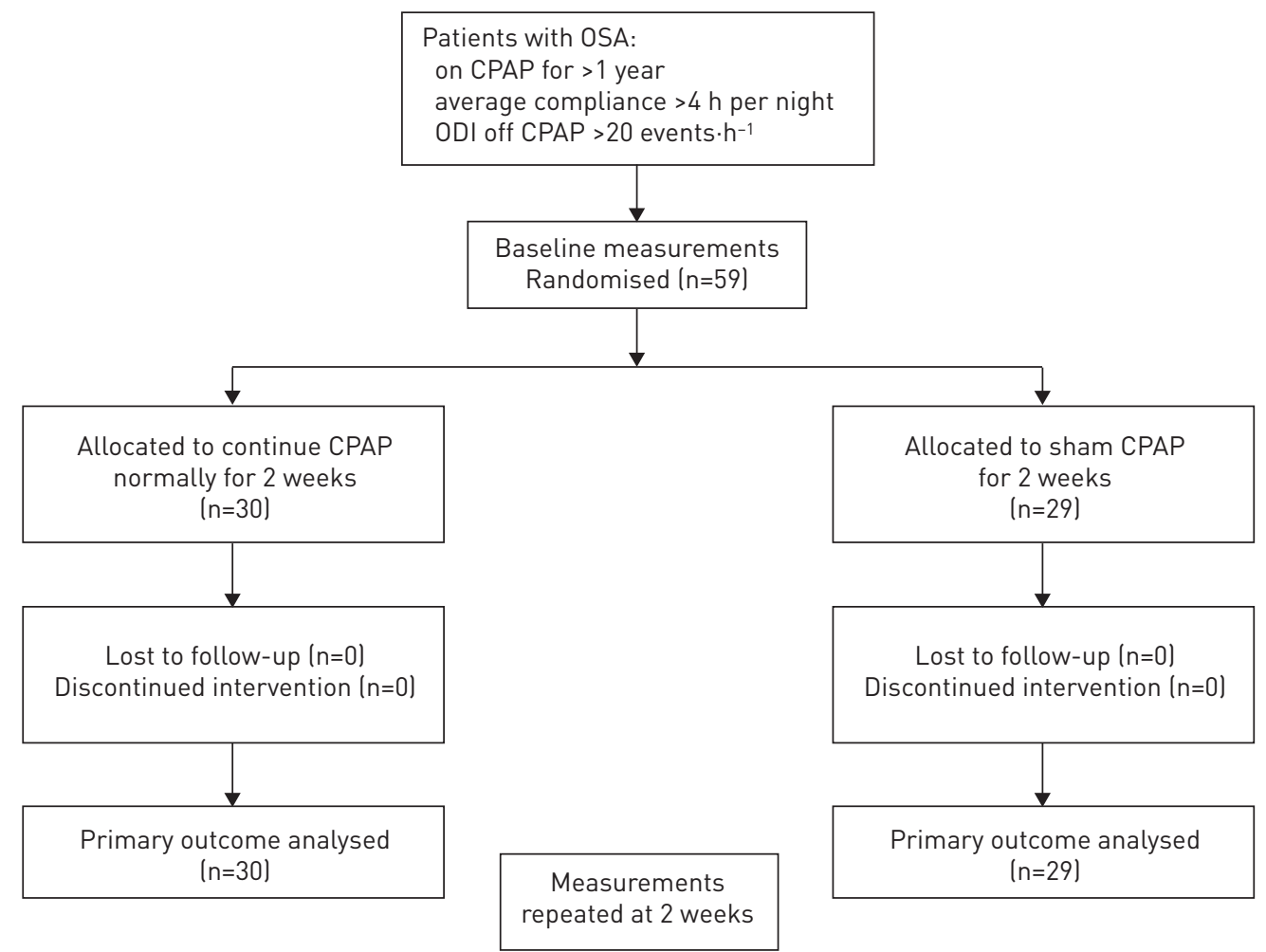

FIGURE 1 Flow chart of study subjects. OSA: obstructive sleep apnoea; CPAP: continuous positive airway pressure; ODI: oxygen desaturation index.

MDA levels, our primary outcome, have been shown to be a sensitive marker of changes in oxidative stress in many clinical and experimental situations, for example following exercise [19], statins [20, 21] and smoking [22]. The 95\% confidence intervals of our "treatment" effect show that we have effectively excluded a difference of $\sim 15 \%$ due to the intermittent nocturnal hypoxia (our study was powered to exclude a change of $\geqslant 20 \%$ with $90 \%$ power). However, we may have missed a rise of $<15 \%$ in MDA.

Because of the unexpected finding of a fall in F2-isoprostanes, we further investigated whether intermittent hypoxia may have caused hypoxic (or ischaemic) preconditioning, which can protect tissues from subsequent ischaemia if the period of prior ischaemia is long enough [14, 23, 24]. This has been hypothesised as potentially occurring in OSA [25-27]. As an exploratory analysis, not in the original protocol, we measured

\section{TABLE 1 Patient baseline characteristics}

\section{Control CPAP withdrawal}

\begin{tabular}{|c|c|c|}
\hline Subjects & 30 & 29 \\
\hline Age years & $59.2 \pm 9.2$ & $62.1 \pm 10.3$ \\
\hline $\mathrm{BMI} \mathrm{kg} \cdot \mathrm{m}^{-2}$ & $34.3 \pm 6.3$ & $35.1 \pm 7.3$ \\
\hline Male:female & $25: 5$ & $23: 6$ \\
\hline Epworth Sleepiness Score & $6.5 \pm 3.7$ & $6.6 \pm 3.5$ \\
\hline Overnight ODI on CPAP events $\cdot \mathrm{h}^{-1}$ & $2.6 \pm 2.4$ & $3.9 \pm 3.8$ \\
\hline Overnight ODI during 4 nights preliminary CPAP withdrawal events $\cdot \mathrm{h}^{-1}$ & $33.4 \pm 14.4$ & $38.7 \pm 16.2$ \\
\hline Morning laboratory systolic blood pressure $\mathrm{mmHg}$ & $133.5 \pm 14.2$ & $125.9 \pm 14.6$ \\
\hline Morning laboratory diastolic blood pressure $\mathrm{mmHg}$ & $83.6 \pm 13.0$ & $80.8 \pm 9.8$ \\
\hline Morning laboratory heart rate beats $\cdot \min ^{-1}$ & $68.7 \pm 8.9$ & $68.3 \pm 9.33$ \\
\hline Statins & $14(47)$ & $11(38)$ \\
\hline Antihypertensive drugs & $17(57)$ & $14(48)$ \\
\hline Smoking status never-smoker:ex-smoker:current smoker & $14: 9: 7$ & $10: 15: 4$ \\
\hline
\end{tabular}

Data are presented as $n$, mean \pm SD or $n(\%)$, unless otherwise stated. CPAP: continuous positive airway pressure; BMI: body mass index; ODI: oxygen desaturation index. 
TABLE 2 Markers of oxidative stress

\begin{tabular}{|c|c|c|c|c|c|c|}
\hline & \multicolumn{2}{|c|}{ Control } & \multicolumn{2}{|c|}{ CPAP withdrawal } & Treatment effect $\#$ & p-value \\
\hline Subjects & \multicolumn{2}{|c|}{30} & \multicolumn{2}{|c|}{29} & & \\
\hline Plasma MDA $\mu \mathrm{mol} \cdot \mathrm{L}^{-1}$ & $1.43 \pm 0.34$ & $1.39 \pm 0.31$ & $1.36 \pm 0.44$ & $1.38 \pm 0.47$ & $-0.02(-0.23$ to +0.19$)$ & $0.86^{+}$ \\
\hline Plasma oxLDL $\mathrm{mU} \cdot \mathrm{L}^{-1}$ & $11.1 \pm 3.72$ & $11.6 \pm 4.85$ & $13.0 \pm 4.20$ & $13.2 \pm 5.02$ & $-0.21(-2.06$ to +1.64$)$ & $0.82^{+}$ \\
\hline Plasma TAC nmol- $\mu \mathrm{L}^{-1}$ & $37.7 \pm 1.71$ & $38.0 \pm 2.31$ & $37.5 \pm 2.37$ & $37.1 \pm 2.14$ & $-0.77(-1.96$ to +0.43$)$ & $0.20^{+}$ \\
\hline
\end{tabular}

Data are presented as $n$, mean \pm SD or mean $(95 \% \mathrm{Cl})$, unless otherwise stated. CPAP: continuous positive airway pressure; ODI: oxygen desaturation index; MDA: malondialdehyde; oxLDL: oxidised low-density lipoprotein; TAC: total antioxidant capacity; F2-ISO: F2-isoprostanes; PBMC: peripheral blood mononuclear cells; RLU: relative light units. \#: treatment effect was controlled for the baseline value of the relevant dependent variable, age, body mass index, statins, antihypertensives and smoking status; " : 13 subjects were included in this analysis (control subjects $n=7$, CPAP-withdrawal subjects $n=6$ ); ${ }^{+}$: nonsignificant.

levels of superoxide dismutase by a commercial ELISA (Abcam, Cambridge, UK; Cat\# ab119520). Superoxide dismutase is an enzyme known to be increased in association with hypoxic preconditioning [28]. The results of this additional analysis are shown in table 3 and indicate a significant rise in SOD of $230 \%$ in the CPAP-withdrawal group, compared to the group continuing on CPAP. This supports the hypothesis that 2 weeks of intermittent nocturnal hypoxia from OSA has in some way increased defences against oxidative stress and thus potentially lessened its effects.

Other studies of OSA or intermittent hypoxia and their possible effects on oxidative stress have recently been well reviewed by BADran et al. [13] and Lavie [29]. Although there are associations between OSA and markers of oxidative stress, such cross-sectional studies are prone to confounding variables and cannot establish a causal relationship. Cross-sectional and uncontrolled studies of CPAP have also shown conflicting results, with some finding a relationship with OSA severity, or improvement with CPAP [30-32], and others not $[18,33,34]$. The only other similarly robust study to ours, by Alonso-Fernández et al. [35], looking at the effect of 12 weeks CPAP versus sham CPAP in a crossover design which recruited 25 newly diagnosed patients with OSA, showed that plasma 8-isoprostane levels were reduced following CPAP by $\sim 40 \%$, and total nitrates and nitrites rose nearly threefold (suggesting increased NO production) compared to control.

Animal studies of OSA have been conducted in a more rigorous fashion with appropriate experimental controls. However, such studies generally use levels of hypoxia far more severe than seen in most patients with OSA, and will also be associated with hyperventilation and hypocapnia, rather than the hypoventilation and hypercapnia seen in OSA (which will tend to promote vasodilation and preserve tissue oxygenation). Such animal studies have found evidence of increased oxidative stress from intermittent hypoxia, for example SAVRANSKY et al. [36] showed that 6 months of intermittent hypoxia in mice quadrupled serum MDA levels compared to control mice. There are animal models more closely simulating OSA, using asphyxia rather than simple hypoxic gas mixtures, but these models have not been used to study oxidative stress.

Hypoxic preconditioning from intermittent hypoxia or OSA has not been as widely studied; this is well reviewed by LAVIE [29]. It is clear that intermittent hypoxia, perhaps via ROS themselves, may also upregulate genes that control antioxidant pathways, such as Nrf2 [37]. The resulting balance of ROS

TABLE 3 Superoxide dismutase: marker of hypoxic preconditioning

\begin{tabular}{|c|c|c|c|c|c|c|}
\hline & \multicolumn{2}{|c|}{ Control } & \multicolumn{2}{|c|}{ CPAP withdrawal } & \multirow[t]{2}{*}{ Treatment effect ${ }^{\#}$} & \multirow[t]{2}{*}{ p-value } \\
\hline & Baseline & 2 weeks & Baseline & 2 weeks & & \\
\hline Subjects & \multicolumn{2}{|c|}{30} & \multicolumn{2}{|c|}{29} & & \\
\hline
\end{tabular}

Data are presented as $n$, mean \pm SD or mean $\left(95 \%\right.$ CI), unless otherwise stated. CPAP: continuous positive airway pressure. ${ }^{\#}$ : treatment effect was controlled for the baseline value of the relevant dependent variable, age, body mass index, statins, antihypertensives and smoking status. 
production versus antioxidant production is likely to be complex and depends on many factors such as hypoxia depth, cycle length and duration; as well as the presence of comorbidities and pre-existing levels of antioxidants. However, in our model, which exactly reproduces the situation in human OSA, albeit for only 2 weeks, it appears that antioxidant capacity dominates. It may be that there was interindividual variation in our patients, with some subjects increasing and some decreasing their levels of oxidative stress markers, leading to no overall average effect on MDA. This is perhaps partially supported by a nonsignificant increase in the standard deviation of the MDA after 2 weeks of CPAP withdrawal, but this effect was not seen in the other markers.

Although we adopted an in vivo human model for the investigation of OSA, with appropriate controls, there are still some limitations. As mentioned above, the intermittent hypoxia only occurred for 2 weeks. It is possible that both shorter and/or longer periods might have produced different results, and might explain the difference between our results and those of the 12-week study by ALONSO-FERNÁndEZ et al. [35]. In measuring one-off blood markers of oxidative stress early in the morning, we have not assessed whether changes might have been missed in markers with very short half-lives. This might explain why the F2-isoprostanes showed changes not reflected in the blood markers, as urine collections will represent a more integrated measure covering the last hours of sleep. In addition, of course, all these measures are indirect and may not fully represent what is happening within the mitochondria and cytosol. Although statins and antihypertensive medications can influence oxidative status, their use was corrected for in the effect-size calculation, and a post hoc analysis found no significant difference in any of the outcome measures dependent on whether these medications were being taken or not. Finally, the patient population studied will not be typical of all patients on CPAP for OSA, as they were selected for greater severity than average, had to be willing to stop CPAP for 2 weeks (which many patients are not prepared to do) and had to demonstrate a rapid return of moderate-to-severe OSA within 4 nights of CPAP withdrawal.

In conclusion, this randomised controlled study of OSA has demonstrated a reduction in a marker of oxidative stress after 2 weeks of intermittent hypoxia, as evidenced by a significant decrease of urinary F2-isoprostanes. A further exploratory analysis showed a rise in plasma superoxide dismutase, which is an antioxidant known to be increased by hypoxic preconditioning. Our findings support the hypothesis that OSA may actually reduce oxidative stress in the short term, and highlight the targets for future investigations.

\section{Acknowledgements}

The authors would like to thank Barbara Winter and Deborah Nicoll (Oxford Respiratory Trials Unit, Churchill Hospital, Oxford, UK) for their dedicated commitment to the running of this trial.

\section{References}

1 Kohler M, Stoewhas AC, Ayers L, et al. Effects of continuous positive airway pressure therapy withdrawal in patients with obstructive sleep apnea: a randomized controlled trial. Am J Respir Crit Care Med 2011; 184: 1192-1199.

2 Kohler M, Craig S, Pepperell JC, et al. CPAP improves endothelial function in patients with minimally symptomatic OSA: results from a subset study of the MOSAIC trial. Chest 2013; 144: 896-902.

3 Pepperell JC, Ramdassingh-Dow S, Crosthwaite N, et al. Ambulatory blood pressure after therapeutic and subtherapeutic nasal continuous positive airway pressure for obstructive sleep apnoea: a randomised parallel trial. Lancet 2002; 359: 204-210.

4 Marin JM, Carrizo SJ, Vicente E, et al. Long-term cardiovascular outcomes in men with obstructive sleep apnoea-hypopnoea with or without treatment with continuous positive airway pressure: an observational study. Lancet 2005; 365: 1046-1053.

5 Kohler M, Stradling JR. Mechanisms of vascular damage in obstructive sleep apnea. Nat Rev Cardiol 2010; 7: 677-685.

6 Kohler M, Pepperell JC, Casadei B, et al. CPAP and measures of cardiovascular risk in males with OSAS. Eur Respir J 2008; 32: 1488-1496.

7 Lavie L, Polotsky V. Cardiovascular aspects in obstructive sleep apnea syndrome - molecular issues, hypoxia and cytokine profiles. Respiration 2009; 78: 361-370.

8 Sert Kuniyoshi FH, Singh P, Gami AS, et al. Patients with obstructive sleep apnea exhibit impaired endothelial function after myocardial infarction. Chest 2011; 140: 62-67.

9 Sitia S, Tomasoni L, Atzeni F, et al. From endothelial dysfunction to atherosclerosis. Autoimmun Rev 2010; 9 : 830-834.

10 Kohler M, Stradling JR. CrossTalk proposal: most of the cardiovascular consequences of OSA are due to increased sympathetic activity. J Physiol 2012; 590: 2813-2815.

11 Lavie L, Lavie P. CrossTalk opposing view: most cardiovascular diseases in sleep apnoea are not caused by sympathetic activation. J Physiol 2012; 590: 2817-2819.

12 Garvey JF, Taylor CT, McNicholas WT. Cardiovascular disease in obstructive sleep apnoea syndrome: the role of intermittent hypoxia and inflammation. Eur Respir J 2009; 33: 1195-1205.

13 Badran M, Ayas N, Laher I. Cardiovascular complications of sleep apnea: role of oxidative stress. Oxid Med Cell Longev 2014; 2014: 985258.

14 Valko M, Leibfritz D, Moncol J, et al. Free radicals and antioxidants in normal physiological functions and human disease. Int J Biochem Cell Biol 2007; 39: 44-84. 
15 Hoffmann MS, Singh P, Wolk R, et al. Obstructive sleep apnea and intermittent hypoxia increase expression of dual specificity phosphatase 1 . Atherosclerosis $2013 ; 231$ : 378-383.

16 Polak J, Shimoda LA, Drager LF, et al. Intermittent hypoxia impairs glucose homeostasis in C57BL6/J mice: partial improvement with cessation of the exposure. Sleep 2013; 36: 1483-1490.

17 Brugniaux JV, Pialoux V, Foster GE, et al. Effects of intermittent hypoxia on erythropoietin, soluble erythropoietin receptor and ventilation in humans. Eur Respir J 2011; 37: 880-887.

18 Katsoulis K, Kontakiotis T, Spanogiannis D, et al. Total antioxidant status in patients with obstructive sleep apnea without comorbidities: the role of the severity of the disease. Sleep Breath 2011; 15: 861-866.

19 Stepanyan V, Crowe M, Haleagrahara N, et al. Effects of vitamin E supplementation on exercise-induced oxidative stress: a meta-analysis. Appl Physiol Nutr Metab 2014; 39: 1029-1037.

20 Moon GJ, Kim SJ, Cho YH, et al. Antioxidant effects of statins in patients with atherosclerotic cerebrovascular disease. J Clin Neurol 2014; 10: 140-147.

21 Antoniades C, Bakogiannis C, Tousoulis D, et al. Preoperative atorvastatin treatment in CABG patients rapidly improves vein graft redox state by inhibition of Racl and NADPH-oxidase activity. Circulation 2010; 122: S66-S73.

22 Lykkesfeldt J. Malondialdehyde as biomarker of oxidative damage to lipids caused by smoking. Clin Chim Acta 2007; 380: 50-58.

23 Park AM, Nagase H, Kumar SV, et al. Effects of intermittent hypoxia on the heart. Antioxid Redox Signal 2007; 9: 723-729.

24 Heusch G, Bøtker HE, Przyklenk K, et al. Remote ischemic conditioning. J Am Coll Cardiol 2015; 65: 177-195.

25 Almendros I, Wang Y, Gozal D. The polymorphic and contradictory aspects of intermittent hypoxia. Am J Physiol Lung Cell Mol Physiol 2014; 307: L129-L140.

26 Ozeke O, Ozer C, Gungor M, et al. Chronic intermittent hypoxia caused by obstructive sleep apnea may play an important role in explaining the morbidity-mortality paradox of obesity. Med Hypotheses 2011; 76: 61-63.

27 Shah N, Redline S, Yaggi HK, et al. Obstructive sleep apnea and acute myocardial infarction severity: ischemic preconditioning? Sleep Breath 2013; 17: 819-826.

28 Chen CF, Tsai SY, Ma MC, et al. Hypoxic preconditioning enhances renal superoxide dismutase levels in rats. J Physiol 2003; 552: 561-569.

29 Lavie L. Oxidative stress in obstructive sleep apnea and intermittent hypoxia - revisited - the bad ugly and good: implications to the heart and brain. Sleep Med Rev 2015; 20: 27-45.

30 Yamauchi M, Nakano H, Maekawa J, et al. Oxidative stress in obstructive sleep apnea. Chest 2005; 127: 1674-1679.

31 Lavie L, Vishnevsky A, Lavie P. Evidence for lipid peroxidation in obstructive sleep apnea. Sleep 2004; 27: $123-128$.

32 Carpagnano GE, Kharitonov SA, Resta O, et al. 8-Isoprostane, a marker of oxidative stress, is increased in exhaled breath condensate of patients with obstructive sleep apnea after night and is reduced by continuous positive airway pressure therapy. Chest 2003; 124: 1386-1392.

33 Svatikova A, Wolk R, Lerman LO, et al. Oxidative stress in obstructive sleep apnoea. Eur Heart J 2005; 26 : 2435-2439.

34 Wali SO, Bahammam AS, Massaeli H, et al. Susceptibility of LDL to oxidative stress in obstructive sleep apnea. Sleep 1998; 21: 290-296.

35 Alonso-Fernández A, García-Río F, Arias MA, et al. Effects of CPAP on oxidative stress and nitrate efficiency in sleep apnoea: a randomised trial. Thorax 2009; 64: 581-586.

36 Savransky V, Bevans S, Nanayakkara A, et al. Chronic intermittent hypoxia causes hepatitis in a mouse model of diet-induced fatty liver. Am J Physiol Gastrointest Liver Physiol 2007; 293: G871-G877.

37 Polotsky VY, Savransky V, Bevans-Fonti S, et al. Intermittent and sustained hypoxia induce a similar gene expression profile in human aortic endothelial cells. Physiol Genomics 2010; 41: 306-314. 